\title{
ESTRIDOR PERSISTENTE
}

\section{PERSISTENT STRIDOR}

Dra. Yazmín A. Márquez R. ${ }^{1}$, Dr. Mario Soto R. ${ }^{2}$, Dr. Raúl Hernández S. ${ }^{3}$, Dr. Luis C. Hinojos G. ${ }^{4}$

1. Médico Residente de segundo año de Neumología Pediátrica. Universidad Autónoma de Chihuahua. Hospital Infantil de Especialidades de Chihuahua.

2. Médico Adscrito y Profesor Titular de la especialidad de Neumología Pediátrica. Universidad Autónoma de Chihuahua Hospital Infantil de Especialidades de Chihuahua.

3. Jefe del Servicio de Neumología Pediátrica y Cirugía de Tórax y Profesor Adjunto de la especialidad de Neumología Pediátrica. Universidad Autónoma de Chihuahua. Hospital Infantil de Especialidades de Chihuahua.

4. Médico Adscrito de Neumología Pediátrica y Profesor Adjunto de la especialidad de Neumología Pediátrica. Universidad Autónoma de Chihuahua. Hospital Infantil de Especialidades de Chihuahua.

\section{ABSTRACT}

Stridor is a musical, often high-pitched sound produced by a rapid, turbulent flow of air through a narrowed segment of the large airways. The cause of stridor can be located anywhere in the extrathoracic airway (nose, pharynx, larynx, and trachea) or the intrathoracic airway (tracheobronchial tree). Stridor may be acute (caused by inflammation/infection or foreign body inhalation) or chronic. It may be congenital or acquired. Various congenital and acquired disorders prevail in neonates, infants, children, and adolescents, and need to be distinguished. Medical history and age of the child, together with physical examination, often allow a presumptive diagnosis. Further tests may be necessary to establish a definite diagnosis, and flexible airway endoscopy is the diagnostic procedure of choice in most circumstances.

Keywords: airway obstruction, stridor, respiratory sounds, bronchoscopy, children.

\section{RESUMEN}

Estridor es un sonido musical, de alta frecuencia, generado por el paso de un flujo de aire turbulento en zonas con calibre disminuido de la vía aérea de mayor tamaño.

La causa del estridor puede estar localizado en cualquier lugar de la vía aérea extratorácica (nariz, faringe, laringe y tráquea) 0 la vía aérea intratorácica (árbol traqueobronquial). El estridor puede ser agudo (causado por inflamación/infección o aspiración de cuerpos extraños) 0 crónica. Puede ser congénita 0 adquirida.

Varios trastornos congénitos y adquiridos prevalecen en neonatos, lactantes, niños y adolescentes, y tienen que ser distinguidos. La historia clínica, edad del niño y examen físico a menudo permiten un diagnóstico presuntivo. Puede requerirse mayor estudio para establecer un diagnóstico definitivo, y la endoscopia flexible de la vía aérea es el procedimiento diagnóstico de elección en la mayoría de las circunstancias.

Palabras clave: obstrucción de las vías aéreas, estridor, ruidos respiratorios, broncoscopia, niños.

\section{INTRODUCCIÓN}

El estridor es un sonido respiratorio de tono alto generado por un flujo rápido y turbulento de aire a través de estrechamientos de vías aéreas de mayor calibre (región supraglótica, laringe, región subglótica y tráquea proximal).

Dado que las vías respiratorias extratorácicas son de menor diámetro durante la inspiración, el estridor suele ser inspiratorio y se vuelve bifásico en caso de estrechamiento grave. Para las vías respiratorias intratorácicas, el diámetro es menor durante la espiración.

Aunque la disminución del lumen de la tráquea proximal puede causar estridor espiratorio, si esto ocurre en las vías respiratorias intratorácicas generalmente da lugar a sibilancias (1).

\section{Correspondencia:}

Dra. Yazmín A Márquez R.

Hospital Infantil de Especialidades de Chihuahua

Av Prolongación Pacheco S/N col Avalos

Chihuahua, México

Correo electrónico: alexjc@gmail.com
El estridor bifásico sugiere una obstrucción severa y fija de la vía aérea a nivel de glotis, subglotis o tráquea superior. Si el estridor se asocia a retracciones, se debe a un estrechamiento significativo de la vía aérea; sin embargo, una disminución repentina en el volumen a veces significa agravamiento de la obstrucción y disminución del movimiento del aire.

El carácter de la voz proporciona información adicional importante; ronquera sugiere una anormalidad de las cuerdas vocales, una voz ahogada con un estridor bajo puede indicar un proceso supraglótico como la epiglotitis (2).

\section{ANATOMÍA DE LA VÍA RESPIRATORIA SUPERIOR}

Durante los primeros 2-5 meses de vida, la lengua del bebé toca todos los bordes de la cavidad oral. Esto y otros factores hace del niño un respirador nasal preferencial; y la obstrucción nasal (especialmente bilateral) puede causar dificultad respiratoria significativa (1).

El hueso hioides del lactante y la laringe están situados más anterior y superiormente que en el adulto. En torno a la edad de 4 meses, la faringe comienza a alargarse y esto puede ir 
acompañado de dificultades de deglución, con una laringe menos protegida de la aspiración. El espacio subglótico, rodeado por el cartílago cricoides, es la parte más estrecha con un diámetro de 5 a $7 \mathrm{~mm}$. Si el tejido conectivo que lo recubre presenta edema causado por inflamación o trauma, un estrechamiento de $1 \mathrm{~mm}$ resultará en una disminución del $75 \%$ del diámetro y, de acuerdo con la ley de Poiseuille y un aumento de 16 veces la resistencia de las vías respiratorias. La luz subglótica es elíptica, y esta forma está determinada por la lámina posterior del cartílago cricoides (1).

\section{HISTORIA CLÍNICA Y EXAMEN FÍSICO}

La historia clínica de un niño con estridor debe incluir historia perinatal y obstétrica: presencia de condilomas maternos, tipo de parto y complicaciones incluyendo distocia del hombro, intubación endotraqueal y su duración, presencia de anomalías congénitas. Se debe caracterizar el estridor: edad de comienzo, variación diurna y relación con alimentación y posición corporal, con el Ilanto, sueño. La evaluación del tipo de estridor (inspiratorio, espiratorio, bifásico) puede proporcionar información sobre la gravedad y el nivel de obstrucción de las vías respiratorias (3). También se debe preguntar por perfil de crecimiento, sospecha de aspiración o ingestión de cuerpo extraño, calidad de voz, síntomas de reflujo gastroesofágico, dificultades de alimentación o síntomas de aspiración, reflujo nasofaríngeo, síntomas asociados tales como retracción, cianosis, 0 apneas, y diagnóstico pulmonar 0 enfermedad neurológica. Se debe obtener antecedentes quirúrgicos, particularmente de cuello o procedimientos cardiotorácicos con riesgo de lesión del nervio laríngeo recurrente.

La alimentación y el crecimiento deben ser evaluados ya que la obstrucción significativa incrementa el trabajo respiratorio, lo que resulta en una falta de ganancia de peso y pobre crecimiento.

Durante la inspección y la auscultación se debe prestar atención a las retracciones (suprasternal, intercostal 0 subcostal), aleteo nasal, cianosis, la posición del paciente y las posibles deformidades sindrómicas (especialmente anomalías craneofaciales) y búsqueda de hemangiomas.

\section{ESTUDIO}

\section{Endoscopia de la vía aérea}

La visualización de la vía aérea está indicada en prácticamente todos los niños con estridor persistente, especialmente cuando el estridor es progresivo 0 asociado con cianosis, apneas, dificultad en la alimentación, o falta de ganancia ponderal. Es importante que el examen de las estructuras supralaríngeas sea antes de aplicar anestesia tópica a la entrada laríngea ya que la lidocaína puede exagerar los hallazgos comúnmente asociados con laringomalacia (1)

En el croup viral, la endoscopia rara vez se indica a menos que no haya respuesta al tratamiento 0 el estridor persista durante más de dos semanas. (3)
Imágenes

En aquellos casos de sospecha de aspiración de cuerpo extraño puede ser de utilidad una radiografía simple del cuello y de tórax, posteroanterior y lateral, en los casos de un objeto radio opaco. En el caso de objetos radiolúcidos se deben buscar cambios indirectos tales como atrapamiento de aire, alteraciones en el parénquima pulmonar, o en la columna traqueobronquial.

Los estudios con contraste de vía digestiva superior pueden ser útiles cuando se sospecha aspiración y ser sugestivos en condiciones tales como una fístula traqueoesofágica de tipo $\mathrm{H} O$ una hendidura laríngea posterior. El trago de bario sigue siendo una excelente técnica para el diagnóstico de un anillo vascular, pero la anatomía exacta de una malformación vascular y su relación con las estructuras adyacentes debe definirse con precisión por resonancia magnética $\mathrm{y} / 0$ tomografía computarizada. Estas últimas técnicas son útiles para confirmar la compresión extrínseca de las vías aéreas, y proporcionan información sobre el alcance de masas tales como hemangiomas en los tejidos blandos circundantes. Además, la endoscopia virtual por tomografía computada puede ser utilizada en la evaluación de estenosis fijas que no se pueden sobrepasar durante la endoscopia.

La ecografía puede ser valiosa en los abscesos parafaríngeos y periamigdalinos y puede proporcionar información valiosa para diferenciar masa quística de sólidos y en la evaluación de las cuerdas vocales (3).

\section{Pruebas de función respiratoria}

La curva de flujo-volumen con obstrucción variable de la vía aérea extratorácica se caracteriza por una meseta en el asa inspiratoria (por ejemplo, traqueomalacia de la tráquea extratorácica) y en un paciente con obstrucción fija se caracteriza por mesetas en las asas inspiratoria y espiratoria (por ejemplo, estenosis subglótica) (Figura 1) (3).

Figura 1. Curva flujo / volumen en obstrucción fija de la vía aérea central.

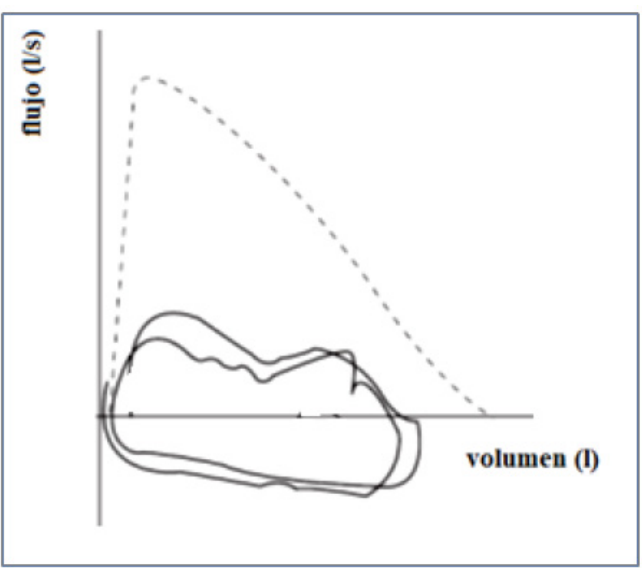

Aplanamiento de las fases espiratoria e inspiratoria 


\section{ETIOLOGÍA}

Según su ubicación anatómica, las causas de estridor se pueden dividir en dos grupos, las que se encuentran a nivel de la nariz y faringe (Tabla 1) y las localizadas en laringe y tráquea (Tabla 2).

Tabla 1. Anomalías más frecuentes de nariz y orofaringe.

\begin{tabular}{l|ll}
\hline \multicolumn{1}{c|}{ Congénitas } & \multicolumn{1}{c}{ Adquiridas } \\
\hline - Atresia / estenosis de Coanas & - Hipertrofia adenoamigdalina \\
Anomalías craneofaciales & - Deformidad septal \\
$\quad$ (Pierre Robin, Treacher Collins) & - Cuerpo extraño \\
- Masas intranasales & \\
- Masas y malformaciones quísticas & \\
(tiroides lingual, malformaciones linfáticas) & \\
Macroglosia & \\
\hline
\end{tabular}

Tabla 2. Anomalías más frecuentes laríngeas y traqueales.

\begin{tabular}{l|l}
\multicolumn{1}{c|}{ Congénitas } & \multicolumn{1}{c}{ Adquiridas } \\
\hline - Laringomalacia & Intubación traumática (estenosis \\
- Parálisis de cuerdas vocales & subglótica adquirida) \\
- Estenosis subglótica congénita & Cuerpo extraño \\
- Traqueomalacia & Infecciones (croup, epiglotitis, \\
- Hemangioma subglótico & papilomatosis laringea) \\
- Membrana laríngea & \\
- Hendidura laríngea posterior & \\
\hline
\end{tabular}

Según su tiempo de evolución se clasifica en agudo y crónico o persistente. Nos referiremos con mayor detalle a este último.

La laringomalacia es la causa más común de estridor en recién nacidos, 45- 75\% de todos los casos de estridor congénito (4). La etiología exacta de la laringomalacia es desconocida, existiendo 3 teorías: anatómica, cartilaginosa y neurológica $(1,5)$. La teoría neurológica, es la mejor apoyada por la literatura, afirma que esta patología puede ser consecuencia de un sistema nervioso central subdesarrollado o anormalmente integrado, particularmente los nervios periféricos y los núcleos del tronco cefálico responsables de la respiración y permeabilidad de las vías respiratorias. Se ha demostrado que el estímulo sensorial necesario para la respuesta motora típica de la laringe es elevado en los lactantes con enfermedad moderada a severa en comparación con aquellos con enfermedad leve (5) La laringomalacia se presenta típicamente con un estridor inspiratorio que aumenta con la alimentación, Ilanto, agitación, posición supina e infecciones del tracto respiratorio superior; y disminuye con el cuello extendido y en la posición prona. Los síntomas comienzan al nacer 0 dentro de las primeras 4 a 6 semanas de vida, con un pico a los 6 a 8 meses y normalmente se resuelve en 12 a 24 meses. Los síntomas asociados más comunes están relacionados con la alimentación, incluyendo regurgitación, emesis, tos y asfixia (4).

El diagnóstico es sospechado por la historia clínica y confirmado por la endoscopia flexible. Es importante examinar los movimientos de las estructuras laríngeas durante la respiración espontánea. El colapso de tejido supraglótico y obstrucción durante la inspiración son el sello distintivo de la laringomalacia. Alteraciones características incluyen elongación y extensión lateral de la epiglotis (forma en omega) que cae posteroinferiormente en la inspiración; aritenoides voluminosas redundantes que prolapsan en la vía aérea en la inspiración; acortamiento de los repliegues aritenoepiglóticos, resultando en el anclaje de las aritenoides a la epiglotis, estrechando así la vía aérea durante inspiración; y el colapso hacia dentro de los cartílagos cuneiformes en la inspiración (6) (Figura 2). Las comorbilidades afectan los síntomas y el curso de la enfermedad; la enfermedad de reflujo gastroesofágico y la enfermedad neurológica son los más comunes.

Figura 2. Tipos de laringomalacia.

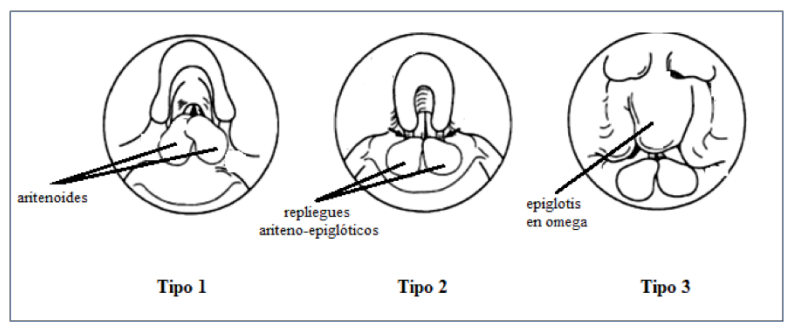

${ }^{*}$ Tipo 1. Prolapso de la mucosa redundante de los aritenoides, que puede introducirse entre las cuerdas en la inspiración.

Tipo 2. Laringomalacia tipo I asociada a repliegues ariepigloticos acortados.

Tipo 3. Laringomalacia tipo II junto con desplazamiento de la epiglotis en omega; puede estar desplazada hacia abajo, pudiendo, incluso introducirse a través de las cuerdas vocales.

La parálisis de cuerdas vocales es la segunda causa más importante de estridor infantil crónico. Puede ser congénita 0 adquirida. La parálisis congénita se manifiesta dentro del primer mes de vida. La parálisis bilateral produce estridor, cianosis y apnea; la parálisis unilateral se presenta con disfonía $(1,6)$. Las causas de parálisis unilateral y bilateral incluyen enfermedad neurológica (18\%), mielomeningocele con malformación de Arnold-Chiari e hidrocefalia (14\%), trauma al nacimiento (19\%), otro trauma (11\%), enfermedad maligna (1\%) y enfermedad familiar (1\%). En el 36\% de la parálisis congénita no se identifica una causa, pero puede existir una asociación con otras enfermedades congénitas (pulmonares, cardiovasculares 0 esofágicas) $(5,6)$. La parálisis adquirida de cuerdas vocales en niños puede ser debido a un trauma quirúrgico (por ejemplo, reparación de una fistula tráqueoesofágica o defectos cardíacos congénitos). Aproximadamente el $50 \%$ de los niños con parálisis bilateral de las cuerdas vocales requiere traqueostomía. Cuando se confirma el diagnóstico de parálisis se recomienda evaluación neurológica y cardiológica pediátrica (8).

La disfunción de las cuerdas vocales puede causar la aparición repentina de estridor y disnea en ausencia de anomalía neurológica identificable. Esta entidad se confunde con asma a pesar de la diferencia entre el estridor inspiratorio, el sonido típico de la obstrucción de las vías respiratorias superiores, y el 
típico sibilante polifónico espiratorio del asma $(9,10)$.

La estenosis subglótica se clasifica en congénita y adquirida. La primera se debe a una canalización incompleta de la zona subglótica y anillo cricoides durante los primeros meses de gestación. La estenosis adquirida se debe a necrosis de la mucosa cricoidea y/o pericondritis de la región subglótica, causadas por intubación endotraqueal prolongada, reiterada 0 traumática. El estridor bifásico es lo característico.

Los quistes laríngeos son una rara malformación laríngea, puede presentarse con obstrucciones graves siendo una emergencia quirúrgica. El diagnóstico se basa en el examen clínico, pero la endoscopia juega un papel diagnóstico y terapéutico. Se clasifican como glóticos, supraglóticos y subglóticos. Zalagh et al los clasificaron según su ubicación: en cuerdas vocales $(58,2 \%)$, pliegues ventriculares $(18,3 \%)$, vallécula $(10,5 \%)$, epiglotis $(10,1 \%)$ y pliegue aritenoepiglótico $(2,2 \%)$. Se postulan dos mecanismos etiopatológicos. Los quistes saculares más grandes son congénitos y se deben a la atresia sacular, mientras que los quistes ductales o quistes de retención, más frecuentes, son adquiridos y resultan de la obstrucción de las glándulas mucosas, que ocurre especialmente durante episodios de infecciones o trauma laríngeo. Se han descrito casos de quistes laríngeos en prematuros que se sometieron a intubación en las primeras horas de vida. Se manifiestan como estridor, disfagia y dificultad respiratoria debido al estrecho conducto laríngeo en los niños (11).

Los hemangiomas subglóticos, son una causa rara de estrechamiento subglótico, experimentan un rápido crecimiento hasta los 12 a 18 meses de edad y luego lentamente involucionan. A los 5 años de edad, el 50\% tienen resolución completa.

Las hendiduras laríngeas ocurren en $1 / 10.000$ nacidos vivos y pueden condicionar disfagia y aspiración. Los niños presentan incremento de secreciones, dificultad para la alimentación, aspiración, retraso de crecimiento y síntomas respiratorios crónicos e infecciones. El diagnóstico definitivo requiere la visualización del área con laringoscopia rígida. (4)

La atresia de coanas involucra la apertura nasal posterior, donde hay obstrucción membranosa (10\%) u ósea $(90 \%)$. Es la anomalía nasal congénita más común (1: 6.000-8.000 nacidos vivos). Los recién nacidos con atresia coanal posterior bilateral tendrán dificultad significativa en la respiración y la alimentación, estridor, cianosis y aspiración. (3) La incapacidad de pasar una sonda a ambos lados de la nariz es característica. La atresia o estenosis unilateral puede pasar desapercibida durante años. La endoscopia flexible y la tomografía computada o resonancia magnética confirman el diagnóstico. El tratamiento definitivo es quirúrgico.

Otras causas menos comunes de obstrucción de vía aérea superior y por tanto estridor son micrognatia, Pierre Robin, macroglosia, anomalías craneofaciales. La micrognatia es una mandíbula inferior pequeña que ocasiona el desplazamiento posterior de la lengua que lleva a la obstrucción faríngea. Se observa predominantemente en la posición de decúbito supino. La secuencia de Pierre Robin se caracteriza por micrognatia, macroglosia, dificultad respiratoria y paladar hendido. Los problemas clínicos dominantes son obstrucción grave de vías aéreas superiores, apnea obstructiva del sueño, y el retraso de crecimiento.

La macroglosia, puede causar obstrucción faríngea.
Puede verse en pacientes con síndrome de BeckwithWiedemann, síndrome de Down, enfermedades del depósito e hipotiroidismo congénito. Los niños con síndrome de Down tienen características fenotípicas que predisponen a obstrucción de la vía aérea superior incluyendo un puente nasal aplanado, boca pequeña e hipofaringe pequeña que junto con la disminución del tono muscular (incluyendo los músculos faríngeos) aumenta la obstrucción proximal de las vías respiratorias. Además, tienen una alta incidencia de laringomalacia, traqueomalacia y estenosis subglótica.

El tiroides lingual es el resultado del fracaso en el descenso de la glándula tiroides desde la base de la lengua a través del conducto tirogloso. El quiste del conducto tirogloso se origina en el remanente del conducto tirogloso. Un quiste se puede llenar con líquido o moco. Si está situado en la base de la lengua y si es lo suficientemente grande, pueden causar obstrucción de las vías respiratorias.

En niños mayores, otras causas raras de estridor pueden incluir anafilaxia, deficiencia de C1 esterasa, hipocalcemia puede producir aducción espasmódica de las cuerdas vocales resultando en la obstrucción de la vía aérea superior y estridor. La hipocalcemia como causa de estridor es rara, y en la edad pediátrica, es más frecuente en los grupos neonatales cuando es generalmente secundario a un hipoparatiroidismo parcial transitorio (12).

\section{Reflujo gastroesofágico}

El ascenso de contenido gástrico a la hipofaringe y laringe puede producir inflamación y edema que compromete aún más la permeabilidad de las vías respiratorias. Existiría asociación entre reflujo gastroesofágico y laringomalacia. El mecanismo es poco claro, sin embargo, el aumento en la presión intratorácica asociada con la laringomalacia puede promover el reflujo del contenido gástrico en el esófago y laringe.

Por otro lado, el reflujo puede promover edema laringofaringea que podría resultar en una disminución de la sensibilidad de los receptores de la mucosa y promover colapso y la disfunción de la deglución y la microaspiración en niños. Parece intuitivo comenzar tratamiento antirreflujo (cambiando la técnica de alimentación, medicación o cirugía) en lactantes y niños con estridor causado por laringomalacia. Sin embargo, no hay prueba de que los síntomas de la laringomalacia mejoran con la terapia antirreflujo $(2,5)$.

\section{CONCLUSIONES}

En niños con estridor, una historia clínica detallada y un examen físico exhaustivo son obligatorios para los pasos diagnósticos adicionales y las intervenciones terapéuticas subsecuentes. Así, se puede realizar un diagnóstico diferencial de las causas más probables de estridor.

La endoscopia de vía aérea establece el diagnóstico en la mayoría de los casos. El paciente debe ser referido a un otorrinolaringologo o neumólogo pediatra para endoscopia flexible de vía aérea superior.

La laringomalacia es la causa más común de estridor congénito y tiene una resolución espontánea sin necesidad de intervención en la mayoría de los pacientes. 
Los autores declaran no tener conflicto de intereses.

\section{REFERENCIAS}

1. Boudewyns A, Claes Y, Van de Heyning P. Clinical practice an approach to stridor in infants and children. Eur $\mathrm{J}$ Pediatr 2010;169:135-141.

2. Escobar ML, Needleman J. Stridor. Pediatr Rev 2015;36;135137.

3. Pfleger A, Eber E. Assessment and causes of stridor. Paediatr Respir Rev 2015; 64-72.

4. Simon D, Price E. Congenital Abnormalities of the Upper Airway. Annals ATS 2016;13(6): 961-962.

5. Daniel M, Cheng A. Neonatal Stridor. Int J Pediatr 2012 ID 859104 doi:10.1155/2012/859104

6. Fauroux B, Pigeot J, Polkey MI, Roger G, Boulé M, Clément A, Lofaso F. Chronic Stridor Caused by Laryngomalacia in Children. Am J Respir Crit Care Med 2001; 164;1874-1878.

7. Olney DR, Greinwald JH Jr, Smith RJH, Bauman NM Laryngomalacia and Its Treatment. Laryngoscope 1999; 109:1770-1776.

8. Weinberger M, Doshi D. Vocal cord dysfunction: a functional cause of respiratory distress. Breathe 2017; 13: 15-21.

9. Hull JA, Backer V, Gibson PE, Fowler SJ. Laryngeal Dysfunction: Assessment and Management for the Clinician. Am J Respir Crit Care Med Vol 194, 2016; 1062-1072

10. Koo DL, Lee JY, Joo EY, Hong SB, Nam H. Acoustic Characteristics of Stridor in Multiple System Atrophy. PLOS ONE 2016:11(4): e0153935.

11. Ahmed $H$, Ndiaye $C$, Barry MW, Thiongane A, Mbaye A, Zemene Y, Ndiaye IC. Rare Cause of Upper Airway Obstruction in a Child. Case Rep Otolaryngol 2017:ID 2017265. doi: 10.1155/2017/2017265

12. Abraham D, Karuvattil R, Fitzpatrick M. Stridor in an 11-yearold child. BMJ Case Rep 2013. doi:10.1136 